\title{
Politizando las ecologías políticas urbanas ${ }^{1}$
}

\section{Politicizing urban political ecologies}

\author{
Erik Swyngedouw ${ }^{2}$ \\ Traducción de Maximiliano Zuñiga Copano
}

\section{Resumen}

El artículo parte de la premisa que es de vital importancia reconocer la rápida tasa de urbanización planetaria como el principal impulsor del cambio ambiental y la creación del Antropoceno. Por lo tanto, este documento no se ocupa mayormente de la cuestión de la naturaleza en la ciudad, como de la urbanización de la naturaleza, entendida como el proceso a través del cual todas las formas de la naturaleza son socialmente movilizadas, incorporadas económica y físicamente, metabolizadas / transformadas, para apoyar el proceso de urbanización. Se argumenta que las transformaciones de nuestro destino urbano y de nuestra naturaleza, están inevitablemente ligadas a una simbiosis metabólica íntima e intensificadora - pero muy contenciosa - caracterizada por patrones socioecológicos extraordinariamente desiguales. La configuración de esta relación metabólica urbana ahora se ha elevado a la dignidad de la preocupación pública mundial, y una búsqueda febril de todo tipo de remedios "sostenibles" eco-profilácticos ha entrado en el vocabulario estándar de los actores gubernamentales y privados. La naturaleza como marco de condicionamiento externo para la vida urbana ha llegado a su fin. La inauguración antropocénica de una naturaleza socio-física, histórica y globalmente urbanizada, obliga a una profunda reconsideración y reescritura de la naturaleza y la urbanización en términos políticos. La cuestión, ya no se trata de llevar los problemas ambientales al dominio de la política urbana, sino de cómo llevar lo político al ambiente urbano.

Palabras clave: Antropoceno, conflicto socio-ecológico, ecología política urbana, política ambiental, teoría urbana.

\begin{abstract}
The paper starts from the premise that it is vitally important to recognize that the rapid rate of planetary urbanization is the main driver of environmental change and the making of the Anthropocene. This paper is, therefore, not so much concerned with the question of nature IN the city, as it is with the urbanization OF nature, understood as the process through which all forms of nature are socially mobilized, economically incorporated and physically metabolized / transformed in order to support the urbanization process. We argue that our urban fate and natures' transformations are irrevocably bound up in an intimate and intensifying metabolic - but highly contentious - symbiosis, one characterized by extraordinarily uneven socio-ecological patterning. The configuration of this urban metabolic relationship has now been elevated to the dignity of global public concern, and a feverish search for all manner of eco-prophylactic 'sustainable' remedies has entered the standard vocabulary of both governmental and private actors. Nature as the externally conditioning frame for urban life has indeed come to an end. The Anthropocenic inauguration of a socio-physical historical and thoroughly globally urbanized nature forces a profound reconsideration and re-scripting of both nature and urbanization in political terms. The question is not any longer about bringing environmental issues into the domain of urban politics, but rather about how to bring the political into the urban environment.
\end{abstract}

Keywords: Anthropocene, environmental politics, socio-ecological conflict, urban political ecology, urban theory.

Recibido el 30 marzo 2018.

Cómo citar: Swyngedouw, E. (2018). Politizando las ecologías políticas urbanas. Investigaciones Geográficas, 56, 153-167. https://doi.org/10.5354/0719-5370.2018.51996

1 Nueva versión traducida al español del capítulo de libro: Swyngedouw, E. (2015). Urbanization and Environmental Futures: Politicizing Urban Political Ecologies. En Perreault T, Bridge G. \& J. McCarthy (Eds.), Handbook of Political Ecology (pp.609-619). London \& New York: Routledge.

2 Department of Geography, University of Manchester, Manchester, United Kingdom. E-mail: erik.swyngedouw@manchester.ac.uk 
“... Urbanismo es el modo de apropiación del medioambiente natural y humano por el capitalismo..." (Debord, 1994, p. 121).

En algún momento del año 2013, la Tierra superó el umbral simbólico de 400 ppm de $\mathrm{CO} 2$ en la atmósfera. En el mismo año, el quinto informe del Panel Intergubernamental para el Cambio Climático (IPCC) concluyó que "la mayoría de los aspectos del cambio climático persistirán durante muchos siglos incluso si se detienen las emisiones de CO2" (IPCC, 2013, p. 27). A pesar de los circos migratorios de las cumbres climáticas de las Naciones Unidas y su deprimente historial de compromisos rotos o de bajo rendimiento, se ha logrado muy poco para reducir las emisiones totales de gases de efecto invernadero. Las emisiones globales de gases de efecto invernadero continúan aumentando año tras año, a excepción del año 2011, posterior a la crisis. Mientras tanto, las ciudades del Norte como en el Sur global, se están asfixiando a medida que la concentración de partículas pequeñas y otras formas de contaminación alcanzan niveles peligrosamente altos.

Ahora hemos entrado verdaderamente en lo que Paul Crutzen (2000) nombró tentativamente el Antropoceno, período geológico que le sucedió al Holoceno. La urbanización planetaria no es solo su forma geográfica, más importante aún, es también el proceso socio-espacial que da forma a la íntima y acelerada fusión de transformaciones y metabolismos sociales y físicos que dieron nombre al Antropoceno (Swyngedouw, 2014, 2017). La urbanización planetaria se refiere al hecho de que cada rincón y grieta de la tierra está ahora, directa o indirectamente, inscrita en asegurar la expansiva reproducción del proceso de urbanización (Brenner, 2013). De hecho, el sustento de la vida urbana existente es responsable del $80 \%$ de las emisiones de gases de efecto invernadero del mundo (Bulkeley \& Betsill, 2005), de la intensificación de la movilización de todo tipo de naturalezas y de la producción de la mayor parte de los desechos del mundo.

Desde esta perspectiva, en este artículo no nos interesa la ciudad como un conjunto denso y heterogéneo de cosas socionaturales acumuladas y cuerpos reunidos en un espacio concentrado, sino más bien, con sus formas particulares de urbanización capitalista como procesos socio-espaciales cuyo funcionamiento se basa en flujos metabólicos socio-ecológicos, a menudo estructurados globalmente, cada vez más largos. Estos flujos no solo unen cosas, naturalezas y pueblos, sino que lo hacen de manera social, ecológica y geográficamente articulada, pero de formas muy desiguales (Angelo \& Wachsmuth, 2015; Cook \& Swyngedouw, 2012). La pregunta clave, por lo tanto, no es sobre qué tipos de naturalezas están presentes en la ciudad, sino más bien sobre la forma capitalista de urbanización de naturalezas: el proceso a través del cual toda clase de "cosas" no humanas se movilizan socialmente, con un guion discursivo imaginado, económicamente inscrito $\mathrm{y}$ mercantilizado, políticamente disputado, regulado institucionalmente y físicamente metabolizado / transformado para producir asociaciones socio-ecológicas que apoyan el proceso de urbanización (Heynen, Kaika \& Swyngedouw, 2006).

Considere, por ejemplo, cuán dependientes son las economías afectivas, supuestamente desmaterializadas, que animan gran parte de la vida social y cultural urbana contemporánea (redes de TI, redes sociales, redes inteligentes, eco-arquitectura, informática y similares) al movilizar un rango de minerales (como Coltan [Columbita-Tantalita]) tras el acaparamiento febril de recursos, a menudo, mediante tácticas de despojo en lugares socioecológicamente vulnerables; en cadenas de producción que están conformadas por metabolismos socio-ecológicos (procesos de producción de materiales e inmateriales) profundamente desiguales $\mathrm{y}$, con frecuencia, deshumanizantes para 
hacerlos útiles en el hardware de ITC, luego de un proceso de reciclado que devuelve gran parte de los desechos electrónicos a las geografías socio-ecológicamente distópicas de las áreas residenciales de los suburbios informales de Mumbai o Dhaka. De hecho, los excesos de la urbanización, desde residuos electrónicos hasta $\mathrm{CO} 2$, se decantan habitualmente en los vertederos socio-ecológicos de las periferias globales de las ciudades del mundo.

La forma capitalista de urbanización planetaria y los procesos socio-ecológicos y político-económicos que animan su desarrollo socio-ecológico combinado y desigual a escala mundial, son ahora generalmente reconocidos como impulsores clave del cambio climático antropogénico y otras transformaciones socio-ambientales como la pérdida de biodiversidad, erosión del suelo, grandes eco-infraestructuras como represas, deforestación, extracción de recursos, la acumulación de plásticos en los diversos ecosistemas del mundo y la minería geológica profunda, la contaminación y la descontrolada mercantilización de todo tipo de naturalezas. Nuestro destino urbano y la transformación de la naturaleza están irrevocablemente vinculados en un íntimo e intenso, pero muy desigual, patrón coreografiado por las relaciones de poder que animan al capitalismo neoliberal. La configuración de esta relación metabólica urbana ha sido en la actualidad elevada a una preocupación pública mundial, y una búsqueda febril por todo tipo de remedios eco-profilácticos ha entrado en el vocabulario estándar de los actores gubernamentales y privados.

De hecho, la búsqueda de una urbanidad socio-ecológica "inteligente" que indague en las cualidades socio-ecológicas del eco-desarrollo, la renovación urbana, la arquitectura sostenible, la gobernanza urbana resiliente, la mercantilización de los servicios ambientales, y el innovador -pero fundamentalmente conforme al mercadodiseño ecológico (Mostafavi \& Doherty,
2010), a menudo se basa en la movilización del trabajo precario y el despojo de la población local de sus recursos y medios de vida (Caprotti, 2014), al tiempo que amplía aún más la movilización de los recursos de la tierra. Este dispositivo técnico-gerencial se ha establecido consensualmente como la frontera entre la arquitectura, la planificación y el diseño urbano, la teoría y la práctica, presumiblemente capaz de salvar tanto a la ciudad como al planeta, mientras asegura que la civilización como la conocemos pueda continuar por un poco más.

La naturaleza como marco de condicionamiento externo para la vida urbana ha llegado a su fin. La inauguración antropocénica de una naturaleza sociofísica histórica y globalmente urbanizada obliga a una profunda reconsideración y reescritura de la naturaleza y la urbanización en términos políticos. La cuestión ya no se trata de llevar las temáticas ambientales al dominio de la política urbana, sino de cómo llevar lo político al medioambiente urbano. En esta contribución, primero exploraremos las formas en que el pensamiento y la investigación urbana han comenzado a incorporar los asuntos políticos dentro de la teoría y la práctica urbana. Se prestará especial atención a las perspectivas de justicia ambiental urbana, por un lado, y a la ecología política urbana por otro. Si bien respaldamos plenamente el extraordinario progreso que se ha logrado en los últimos años, insistiremos, en una segunda parte, en que la ecología política urbana debe tomar la cuestión de "la política en la ecología política" mucho más en serio. Una serie de recomendaciones para una ecología política urbana politizada concluirá el capítulo.

\section{Urbano natural}

El proceso de urbanización, entendido como una parte constitutiva del predicamento socio-ecológico del mundo, estaba en primer plano en la década de 1970 como parte de una preocupación más amplia en conjunto con el deterioro de las 
condiciones ambientales. Mientras que las voces de los visionarios eco-urbanos como Murray Bookchin pasaron en gran medida desapercibidas (White, 2008), el gran llamado maltusiano sobre el anticipado agotamiento de los recursos, pronosticado por los Límites de Crecimiento (Limits to Growth) del Club de Roma, aumentando el espectro de la escasez inmanente en la naturaleza, realmente consiguió que las elites globales se preocuparan por las posibilidades supuestamente débiles para sostener la acumulación capitalista durante mucho más tiempo, y señalaron a la urbanización, como el principal culpable del acelerado agotamiento de los recursos en el mundo (Meadows, Meadows, Randers y Behrens III, 1972). Sumado a esto, el movimiento ambientalista, particularmente activo en la lucha contra el uso de energía nuclear en el Norte Global, y la hiperurbanización en el Sur Global, impulsó los asuntos ambientales a la cima de la agenda de política urbana. El pensamiento y la práctica urbana siguieron su ejemplo. Los académicos y activistas urbanos comenzaron a analizar la urbanización de la naturaleza como un proceso continuo de des-territorializacion y re-territorializacion de los flujos circulatorios metabólicos socio-ecológicos, organizados a través de relaciones sociales predominantemente capitalistas sostenidas por conductos y redes socio-físicas de gestión privada o pública (Swyngedouw, 2006), nutrido por imaginarios particulares de lo que es o debería ser la naturaleza.

Bajo el capitalismo, siguiendo el argumento, la movilización y transformación de las 'cosas' no humanas en una forma mercantilizada bajo el ímpetu de la circulación y la acumulación de capital dan forma a estos procesos socio-ecológicos y convierten a la ciudad en un proceso socioambiental metabólico que se extiende desde el entorno inmediato hasta los rincones más remotos del mundo. A través de esta lente conceptual, la urbanización se ve como un proceso de metabolismos socio-ambientales, geográficamente dispuestos, que fusionan lo social con lo físico. Al hacerlo, se produce una urbanidad cyborg que enmarca distintas formas físicas con relaciones culturales y socioeconómicas de maneras socioecológicas geográficamente muy desiguales (Gandy, 2005; Swyngedouw, 1996).

Un proliferante cuerpo de trabajo académico comenzó a explorar, tanto empírica como teóricamente, cómo la urbanización y sus habitantes humanos y no humanos en todo el mundo están vinculados a través de redes socio-tecnológicas, flujos de materia y relaciones sociales de poder para la extracción, circulación y eliminación de materias como el agua (Swyngedouw, 2004), energía (Bouzarovski, 2014, Verdeil, 2014), grasa (Marvin \& Medd, 2006), productos químicos y desechos electrónicos (Pellow, 2007), alimentos (Heynen, Kurtz, \& Trauger, 2012), residuos domésticos (Armiero, 2014; Njeru, 2006), infraestructuras (Graham \& Marvin, 2001; Monstadt, 2009), o buques redundantes (Buerk, 2006; Hillier, 2009). Apoyándose en el proceso metabólico de actores socio-naturales menos visibles, pero poderosamente importantes, Ali \& Keil (2008) mapearon cómo la epidemia del SARS (Síndrome respiratorio agudo y grave) desafió a las redes globales de gobernanza urbana. Bulkeley \& Betsill (2005) buscaron las raíces urbanas del $\mathrm{CO}_{2}$, y Robbins (2007) reconstruyó las redes mundiales de producción, contaminación y desechos tóxicos, que sostienen el insaciable impulso de nutrir los céspedes "verdes" que alimentan el sueño de la clase media de los suburbios estadounidenses.

Una serie de emocionantes monografías urbanas exploraron las dinámicas político-ecológicas que soportaban la producción histórico-geográfica de ciudades particulares. Por ejemplo, la monografía seminal de William Cronon (1991), Nature's Metropolis, reescribe el proceso de urbanización de Chicago mediante una examinación de cómo la producción de trigo y cerdo dio forma al 
proceso de transformación metabólica y de expansión espacial de la ciudad y vinculó a Chicago con su interior, a otras ciudades y al mercado global. Brechin (2001), narra cómo las elites de San Francisco hurgaron en la naturaleza en busca de la ganancia y el poder terrenal, mientras que en Concrete and Clay, de Matthew Gandy (2002), este se aproxima a la arqueología del proceso de urbanización de Nueva York como una construcción político-ecológica. En algunos estudios, el agua se convirtió en una entrada emblemática en la excavación de flujos socio-ecológicos. City of Flows de Maria Kaika (2005) considera las relaciones culturales, socioeconómicas y políticas a través de las cuales la circulación del agua en Atenas y otras ciudades se organiza y reorganiza durante la modernidad.

En Social Power and the Urbanization of Nature (Swyngedouw, 2004) se examina la relación entre la urbanización planetaria y la transformación de la naturaleza a través del lente del agua de Guayaquil, mientras que Karen Bakker (2003), sigue el flujo del agua a través de las políticas de privatización de Inglaterra y Gales; y Masjuan, March, Domene, \& Saurí (2008) exploran las dinámicas, conflictos y luchas político-ecológicas en torno al suministro de agua urbano de Barcelona. También vale la pena señalar cómo en la distópica Dead Cities and other Tales de Mike Davis (2002) excavan las ecologías peculiares de las ciudades que no deberían estar donde están, y el majestuoso estudio de Freidberg (2004) de cómo los frijoles verdes unen a las ciudades africanas con París y Londres, explorando, cómo la urbanización es efectivamente sostenida por redes socioecológicas planetarias y relaciones impulsadas por dinámicas particulares de urbanización y vida urbana.

Las narrativas anteriores en ecología política urbana e investigaciones afines demostraron, de diversas maneras y desde una variada gama de perspectivas teóricas, cómo el tema de la materia se convierte en un momento activo en las transformaciones político-ecológicas que dan forma a la urbanización planetaria. Estos autores han argumentado convincentemente que el proceso urbano tiene que ser teorizado, entendido y gestionado como un proceso socio-natural que va más allá de la mediación tecno-gerencial de las relaciones socio-ecológicas urbanas. $\mathrm{Al}$ hacerlo, contribuyeron a deslegitimar las perspectivas dominantes del siglo XX sobre la ciudad que ignoraba la naturaleza, sin caer en el callejón sin salida del fetichismo de la naturaleza o el determinismo ecológico. Además, al trascender la división binaria entre naturaleza y sociedad, la perspectiva del metabolismo urbano ha demostrado que los procesos socio-ecológicos son intensamente políticos y politizados, y confirmó que la teoría urbana sin naturaleza no puede ser más que incompleta.

Sin embargo, este cuerpo de pensamiento ha prestado relativamente poca atención a las posibilidades políticas que tales entendimientos renovados de urbanización podrían aportar, o a imaginar futuros posibles radicalmente diferentes para los conjuntos socio-ecológicos urbanos que son una parte integral de las movilizaciones radicalmente politizadas. Por lo tanto, aunque ahora podamos rastrear, trazar, seguir y narrar las múltiples líneas y flujos socio-ecológicos que dan forma al proceso urbano globalizador, se ha dicho muy poco sobre cómo producir constelaciones urbano socio-ecológicas alternativas más equitativas y habilitantes. En lo que sigue, exploraremos brevemente dos perspectivas que han galvanizado un pensamiento y una práctica más politizada en torno a la cuestión ambiental urbana.

\section{Justicia ambiental urbana: la distribución de los daños ambientales}

La perspectiva de la Justicia Ambiental Urbana (JAU) abre una visión politizante en la que las características de distribución desigual del metabolismo urbano ocupan un lugar central. La JAU es sensible a los 
procesos conflictivos y cargados de poder de la urbanización de la naturaleza a través de arreglos tecno-gerenciales dirigidos por la élite (Walker, 2012). Originada en los Estados Unidos, la justicia ambiental urbana surgió como un concepto normativo y como un movimiento social, sostenido por nuevos conocimientos sobre la distribución altamente desigual de "bienes" y "males" ambientales en la ciudad. El trabajo inicial en la década de 1980 ya había comenzado a reconocer que los vecindarios pobres, a menudo predominantemente afroamericanos, estaban ubicados de manera abrumadora en áreas caracterizadas por condiciones ambientalmente peligrosas (Bullard, 1990). Se encontraron significativas correlaciones positivas entre la presencia de vertederos tóxicos, las instalaciones de procesamiento de desechos, la contaminación del suelo, los productos químicos peligrosos o la ausencia de zonas verdes, por un lado, y las concentraciones de hogares de bajos ingresos, por el otro. En otras palabras, la distribución espacial de los bienes y males ambientales reflejaba la distribución socioespacial del poder político, la riqueza y los ingresos (Schlosberg, 2007).

La justicia ambiental urbana se definió y entendió como una cuestión de (in)justicia distributiva, coreografiada y estructurada por las relaciones altamente desiguales de poder político y económico a través de las cuales se toman las decisiones sobre las condiciones de distribución ambiental. Se hace énfasis en las posiciones sociales y económicamente desiguales, los modos de reconocimiento y las capacidades de los diferentes habitantes urbanos en la maquinaria de toma de decisiones política y económica urbana que asigna la distribución de bienes y males ambientales en toda la ciudad, lo que demuestra que la partición de los "bienes" benefician principalmente a las elites urbanas, mientras que los "males" ambientales se reducen a las áreas donde viven los impotentes y los marginados. La partición de lo sensible en el orden policial, como lo llamaría Jacques Rancière
(2000), se manifiesta, entre otras cosas, en configuraciones socio-ecológicas desiguales con relaciones altamente estratificadas de acceso a "bienes" ambientales o la exposición a "males". Quedó claro que las vidas urbanas sostenibles son principalmente el privilegio de los ricos, y que los paraísos ambientales se sustentan en el deterioro de las condiciones socioecológicas en otros lugares.

Las exploraciones más recientes de las injusticias ambientales urbanas han extendido el enfoque anterior sobre la raza a otras categorías sociales como género, clase, edad, capacidad y escala geográfica (Walker, 2009). No obstante, el énfasis de la JAU permanece claramente en el primer plano de las nociones liberales de justicia procesal y distributiva como equidad, y expresando una forma distinta de NIMBY (Not in my Back Yard). La JAU se ocupa principalmente de los procedimientos a través de los cuales se dividen y distribuyen las tecnologías ambientales, las infraestructuras y los servicios en toda la ciudad, destacando el patrón social altamente desigual de las calidades y peligros ecológicos. Sin embargo, esta perspectiva tuvo éxito en la socialización de la naturaleza y la ecología mediante la excavación de los intrincados mecanismos a través de los cuales la naturaleza, los procesos ecológicos y las condiciones socioambientales de la ciudad están altamente entretejidos en formas profundamente injustas. Esto demuestra cómo la partición de cosas y procesos socio-ambientales a través de prácticas institucionales formales y sociopolíticas informales es completamente no igualitaria, lo que abre la posibilidad de una interrupción política en el sentido de Rancière. La distribución desigual queda grabada en el paisaje urbano a través de una combinación de procedimientos de toma de decisiones altamente elitistas, por un lado, y su incorporación a la arquitectura de las infraestructuras eco-tecnológicas y tecnologías, por el otro. No obstante, la JAU tiende a ser sintomáticamente silenciosa 
acerca de las maneras particulares en que las formas políticas de poder se entrelazan con las modalidades concretas a través de las cuales la naturaleza se inscribe en los procesos de circulación y acumulación de capital. Su enfoque basado en el lugar también debe aumentarse en consideración de los conjuntos y flujos metabólicos globales que producen un cambio socioecológico desigual y combinado.

Ecología política urbana: reafirmando la producción capitalista de la urbanización planetaria

Mientras que la JAU se enfoca principalmente en patrones de injusticia socio-ecológica dentro de la ciudad, la Ecología Política Urbana (EPU) cambia la mirada interpretativa hacia las desigualdades socio-ecológicas incorporadas y moldeadas por la producción y reproducción de la propia urbanización capitalista (Keil, 2003, 2005). Bajo el capitalismo, una amplia gama de naturalezas diferentes se enrolan cada vez más en los circuitos de acumulación de capital a través de los cuales ambos se transforman y son re- y de-territorializados. Este es un proceso socio-metabólico mediante el cual la materia física, como el agua o las vacas, se transforma en productos utilizables, concedidos y comerciables" (Coe, Kelly \& Yeung, 2007, p.161). Desde esta perspectiva, 'Naturaleza' como el nombre colectivo y homogeneizado para todo tipo de cosas, organismos y procesos no humanos, no existe, sino que existe una colección muy diversa y en constante cambio de todo tipo de embrollos no humanos que se son producidos histórica y geográficamente de maneras específicas y decididamente urbanizadas. Es tal conceptualización la que llevó a David Harvey (1993, p. 28; 1996, p.186), por ejemplo, a argumentar que "no hay nada antinatural en la ciudad de Nueva York".

La EPU es decididamente anti-maltusiana. En contraste con el espectro demográfico cargado de fatalidad de los límites maltusianos de la base de recursos de la tierra y la amenaza pendiente de la escasez absoluta, la ecología política urbana considera la escasez como producida socio-ecológicamente a través de los imperativos gemelos de la "acumulación por sentido de la acumulación", por una parte y las fuerzas del "mercado" como instrumentos naturalizados y privilegiados para la asignación social y la distribución de naturalezas (transformadas) en el otro. Además, la EPU rechaza el imaginario apocalíptico que habitualmente acompaña los intentos de poner en el primer plano político una preocupación pública con la naturaleza como inherentemente despolitizante y reaccionaria (Swyngedouw, 2013a, b). Para la EPU, la catástrofe socio-ecológica ya está presente y refleja los patrones socio-ecológicos combinados y desiguales producidos por la forma específicamente capitalista de la globalización de los metabolismos urbanos.

De hecho, una configuración políticoeconómica, usualmentellamada capitalismo, cuya "sustentabilidad" es predicada a partir del crecimiento por el bien del crecimiento, necesariamente se topa con los límites físicos y sociales de sus propias condiciones previas de existencia, lo que desencadena dinámicas continuas y altamente desiguales de reestructuración y transformación socioecológica generalizada. Más importante aún, tales ambientes socio-físicos urbanos producidos encarnan y reflejan el poder desigual y las condiciones de vida socioecológicas asimétricas asociadas inscritas en los metabolismos socio-ecológicos. La "Escasez" o la "desintegración socioecológica" residen, por lo tanto, no en la Naturaleza sino en las modalidades socialmente construidas y totalmente contingentes de su inclusión espacial y socio-ecológicamente variada dentro de los circuitos de urbanización de la circulación y acumulación de capital. La producción de medioambientes urbanos y los "vehículos metabólicos" (como las infraestructuras de todo tipo, las condiciones técnicas 
que permiten el flujo y la metabolización de, entre otros, energía, alimentos, información, cuerpos y cosas) que aseguran su funcionamiento están mediados por acuerdos institucionales y de gobierno que a menudo son nominalmente democráticos, pero que sin embargo están necesariamente profundamente comprometidos a asegurar la expansión ininterrumpida del proceso de circulación de capital (Virilio, 1986). Los vehículos metabólicos son las infraestructuras duras y blandas a través de las cuales se transforma la materia no humana, y expresan en su funcionamiento técnico-político múltiples relaciones de poder en las que los actores sociales se esfuerzan por crear y defender entornos socio-físicos que sirven a sus intereses y satisfacen sus deseos. Es precisamente esta articulación entre el estado, la clase y la traducción ambiental la que vuelve a los procesos socio-ecológicos urbanos, incluida la cuestión de la sostenibilidad, altamente conflictivos y que estén sujetos a una intensa lucha política y social. Considere, por ejemplo, cómo la rebelión urbana que envolvió a Turquía con una intensidad rara vez vista en el verano de 2013 se desató de manera emblemática a partir de un conflicto sobre un parque y algunos árboles en la Plaza Taksim de Estambul. O cómo las cumbres climáticas se enfrentan con protestas callejeras cada vez más intensas.

La urbanización de la naturaleza es decididamente multi-escalar y espacialmente interconectada en una red de manera extendida. Los acuerdos de gobernanza multi-escalar, desde la Agenda 21 hasta el protocolo de Kioto, sugieren cómo se articula el alcance global de los procesos de transformación socio-ecológica con los conjuntos de gobernanza a múltiples escalas, cada uno de los cuales expresa particulares relaciones de poder mediante las cuales las luchas por el control, el acceso y la transformación de la naturaleza y la distribución de bienes y malos ecológicos se negocian cuidadosamente y se cuestionan intensamente. Desde esta perspectiva político-ecológica, las condiciones ecológicas urbanas y las configuraciones de su gobernanza nunca son solo locales, sino que están vinculadas a procesos que operan en diversas ecologías en todo el mundo. Tales enfoques político-ecológicos urbanos ponen en primer plano el núcleo político del cambio y la transformación ambiental e insisten en la naturaleza fundamentalmente política de los modos de organización sociotécnica de la transformación metabólica de la naturaleza.

En resumen, la ecología política urbana se preocupa del proceso político democrático y emancipador a través del cual se lleva a cabo una transformación ecológica políticamente integrada. En lugar de invocar una noción normativa de justicia ambiental o de una naturaleza idealizada (equilibrada), la EPU insiste en centrarse en las realidades de la presumida igualdad política democrática en los procesos de toma de decisiones que organizan la transformación socioecológica y coreografían la gestión de los comunes. Al hacerlo, la atención pasa de una perspectiva tecno-gerencial o ética a una posición decididamente política, articulada en torno a la noción de igualdad, que considera que el dilema ecológico está inexorablemente asociado con la actuación política democrática, y que se centra en las condiciones fundamentalmente politizadas, a través de las cuales se producen las naturalezas (Swyngedouw, 2014).

\section{Politizando la ecología política de la urbanización planetaria}

A pesar del extraordinario salto hacia adelante en la comprensión crítica de la condición ambiental urbana y la atención consensual a las ecotecnologías inteligentes y sustentables, las condiciones ecológicas globales continúan deteriorándose a un ritmo alarmante a medida que se intensifica la urbanización planetaria. Esta es una verdadera situación paradójica que solo puede volverse legible en términos estrictamente ideológicos. 
Slavoj Žižek (2008) plantea que a pesar de que sabemos muy bien (el problema ecológico en el que nos encontramos), seguimos actuando como si no supiéramos. Mientras que las elites tecno-gerenciales intentan desesperadamente micro- $y$ macro-ingenierizar las condiciones socioecológicas de manera que permitan sostener el crecimiento económico indefinidamente en el futuro y convertir las tecnologías ambientales en una estrategia de acumulación "verde", la profundidad y el alcance de la degradación ambiental galopa más allá en lo que Calder Williams (2011) llama un apocalipsis desigual y combinado.

También se vuelve claro que el primer llamado de los ecologistas, tomando prestado del comunista italiano del siglo $\mathrm{XX}$, Amadeo Bordiga, que "cuando el barco se hunde, los pasajeros de primera clase también se ahogan", es evidentemente falso. Los pasajeros urbanos de primera clase de la tierra se encuentran ocupados construyendo embarcaciones de rescate trans-planetarias, mientras los refugiados ecológicos y políticos se ahogan en el Mediterráneo, y muchos otros continúan viviendo en las proliferantes tierras baldías de sus degradantes entornos socioecológicos.

La urbanización planetaria, que se desarrolla a través de la universalización de la mercantilización y la acumulación de naturalezas dentro de una configuración política neoliberalizante, acelera el proceso de apocalipsis ecológico combinado y desigual, uno sostenido cada vez más por la promesa mítica de la sostenibilidad tecnológicamente mediada y las formas post-democráticas de gobernanza consensuada que no toleran la disidencia radical o la búsqueda de verdaderas alternativas político-ecológicas. Los esfuerzos tecno-gerenciales despolitizantes que caracterizan los modos dominantes de gobierno ambiental suturan el paisaje ideológico, y excluyen los modos más políticamente fundamentados de producir un modo socio-ecológico más igualitario de gobernar y transformar los comunes urbanos (Swyngedouw, 2009, 2018). Transgredir este 'punto muerto' entre los peligros reales y actuales de la urbanización planetaria socio-ecológica combinada y desigual, por un lado, y el impotente actuar de la gestión sustentable post-democrática de los recursos y las personas por el otro, exige un compromiso intelectual y político serio con algunas de las condiciones más difíciles e intratables en que se encuentran nuestras ciudades.

Como se argumentó en otra parte (Swyngedouw, 2010, 2018), las preocupaciones consensuadas, como la "sostenibilidad", nutren una postura políticamente reaccionaria. Son una expresión del proceso actual de postpolitización y post-democratización, que se organiza en torno a distintos gestos biopolíticos. La post-politización se refiere a una política en la que la planificación y la intervención tecno-gerencial, la gestión de expertos y la administración bio-política desplazan la disensión o las disputas y luchas ideológicas. Dichos acuerdos de post-politización señalan un estrechamiento profundo de las luchas agonistas democráticas sobre el contenido y la dirección de la vida socio-ecológica, e instituyen un espacio público donde el terreno de la política se ha reducido a la formulación de políticas en las que el conocimiento experto, la intermediación de intereses y la administración a través de la gobernabilidad ha comenzado a reemplazar el debate, la disensión y el encuentro agonístico (Marquand, 2004).

El debate y los argumentos contenciosos se limitan a cuestiones de gestión tecnogerencial, donde el marco neoliberal del desarrollo dirigido por el mercado y centrado en el crecimiento no puede ser cuestionado legítimamente. Este acuerdo consensual despolitizado se organiza a través de instituciones de gobernanza administrativa post-democráticas que están reemplazando cada vez más a las instituciones políticas de gobierno 
(Crouch, 2004) y están insertas en un orden político-económico neoliberalizador geográficamente variado, pero ampliamente naturalizado. Estos argumentos apuntan a la importancia vital de lidiar con este proceso de post-politización y de moverse desde políticas urbanas del medio ambiente a la urbanización de las políticas ambientales. Si el objetivo de la política, incluidas las normas y políticas urbanas, el diseño, la planificación y la arquitectura de la ciudad, es una intervención que pueda cambiar el ordenamiento socio-ambiental dado y la partición en una dirección determinada, dicha intervención a menudo constituye un acto violento, en el sentido que borra lo que está allí (al menos en parte) para erigir algo nuevo y diferente.

El punto central es reconocer que los actos políticos son intervenciones singulares que producen arreglos y entornos socioecológicos particulares, y al hacerlo, excluyen la posibilidad de que surjan otros. Una intervención permite la formación de ciertos conjuntos socio-ecológicos mientras que a su vez cierra otros. La violencia inscrita en tal elección debe ser plenamente reconocida. Por ejemplo, uno no puede tener simultáneamente una ciudad verdaderamente neutral en carbono y permitir una movilidad ilimitada en base a automóviles. Son mutuamente excluyentes. Menos aún puede producirse un futuro urbano igualitario, democrático, solidario y ecológicamente sensible sin marginar o excluir a quienes insisten en la apropiación privada de los bienes comunes de la tierra y su movilización para la acumulación, el enriquecimiento personal y la transferencia hereditaria de recursos acumulados.

Tales encuentros violentos siempre constituyen un acto político, uno que puede ser legitimado solo en términos políticos, y no a través de una legitimación externa que reside en una fantasía de la naturaleza o la sostenibilidad. Cualquier acto político es uno que reordena las coordenadas y patrones socio-ecológicos, reconfigura relaciones socio-ecológicas desiguales (mientras excluye a otros), a menudo con consecuencias imprevistas o imprevisibles. Tales intervenciones que expresan una elección $\mathrm{y}$ toman un partido son invariablemente de alguna manera exclusivas. Cualquier orden instituido, incluido uno liberal-democrático, produce sus propias desigualdades. Esto es precisamente lo que la teoría urbana crítica ha explorado y analizado con éxito en las últimas décadas. Esto sugiere que existe una brecha o abismo irreductible entre lo democrático, como lo político dado sobre la presunción de la igualdad de todos, por una parte, y las formas instituidas / institucionalizadas de elaboración de políticas que suspenden invariablemente esta igualdad axiomática. En otras palabras, cualquier forma de formulación de políticas es hasta cierto punto oligárquica. Esta brecha entre "política" y "lo político" necesita ser completamente respaldada.

Lo más importante, es que enfrenta a aquellos que están empeñados en mantener la trayectoria actual que produce un apocalipsis socio-ecológico desigual y combinado radicalmente en contra de aquellos que prefiguran una producción inclusiva e igualitaria de los bienes comunes socio-ecológicos urbanos. En lugar de invocar una noción normativa de justicia ambiental o de una naturaleza idealizada (equilibrada), nuestra perspectiva insiste en centrarse en las realidades desiguales de la presunta igualdad política democrática en los procesos de toma de decisiones que organizan la transformación socio-ecológica y coreografían la gestión de los comunes. Al hacerlo, la atención pasa de una perspectiva tecno-gerencial, físico-ecológica o ética a una posición decididamente política articulada en torno a la noción de igualdad - que considera que el dilema ecológico está inexorablemente asociado con la acción política democrática y se centra en las condiciones fundamentalmente politizadas a través de las cuales se producen las naturalezas (Swyngedouw, 2014).

Si bien una política democrática pluralista 
insiste en la diferencia, el desacuerdo, la apertura radical y la exploración de múltiples futuros posibles, la intervención espacialecológica concreta es necesariamente sobre el cierre relativo (para algunos), la elección definitiva, la intervención singular $y$, por lo tanto, cierta exclusión y en ocasiones incluso derechamente el silenciamiento. Por ejemplo, la explotación de arenas bituminosas y el fracking no pueden coincidir con una política climática digna de su nombre. La justicia climática requiere un proceso que mantenga el carbón en el agujero, el petróleo en el suelo y la arena bituminosa en la tierra. Esto prohibiría estrictamente las prácticas dominantes y produciría efectos distributivos extraordinarios que solo se pueden atender políticamente. Si bien las políticas democráticas tradicionales se basan en principios mayoritarios, la perspectiva democrática-igualitaria insiste en poner en primer plano la equidad y la solidaridad socio-ecológica como el gesto fundacional para un futuro urbano verde. Por lo tanto, la politización democrática del medio ambiente se convierte en una cuestión de mejorar el contenido político democrático urbano de la construcción socio-ambiental mediante la identificación de estrategias mediante las cuales se puede lograr una distribución más equitativa del poder social y un modo más igualitario de producir naturalezas urbanas.

Esto requiere el fomento de procesos que permitan la producción de espacios de democratización (por ejemplo, espacios para la enunciación de disputas agonistas) como fundación y condición para una mayor cantidad de arreglos socioecológicos urbanos más igualitarios, $y$ el nombramiento de futuros socioecológicos ega-libertarios positivamente incorporados que son inmediatamente realizables. El agonismo se refiere aquí al proceso mediante el cual las posiciones de oposición entre enemigos se articulan y organizan como un encuentro de oposición entre adversarios (Mouffe, 2013). En otras palabras, las ecologías urbanas igualitarias consisten en demandar lo imposible y realizar lo improbable, a menudo frente a una oposición radical y poderosa, y este es exactamente el desafío que plantea el Antropoceno (Swyngedouw \& Ernstson, 2018). En resumen, la politización del medio ambiente es predicada sobre el reconocimiento de la indeterminación de la naturaleza, la división constitutiva de las personas, la demanda democrática incondicional de igualdad política y la posibilidad real de la inauguración de futuros socio-ecológicos urbanos públicos y colectivos que expresan las presunciones democráticas de libertad e igualdad.

Por último, el desafío intelectual planteado por las condiciones socio-ambientales conformadas por la urbanización planetaria debe ser el extender el imaginario intelectual y los poderes del pensamiento y la práctica para superar el impasse cultural contemporáneo identificado por Jameson (2003) "es más fácil imaginar el final del mundo que los cambios en el orden ecocapitalista y sus inequidades" (p. 76). Este es el coraje del intelecto que ahora se requiere más que nunca, un coraje que nos lleva más allá de los límites impotentes de un discurso de sostenibilidad y deja intactas las dispares, pero decididamente urbanizadas, dinámicas socio-ecológicas existentes. Es un coraje que traza nuevas avenidas politizadas para producir una nueva urbanidad común. Por lo tanto, hay una tarea urgente por delante para profundizar en los complejos vínculos entre la politización del discurso y la práctica, la gestión ecológica post-política y la reproducción de las desigualdades socioecológicas ambientales.

Es necesario hacer preguntas sobre qué visiones de la naturaleza y qué relaciones socio-ambientales se están promoviendo, qué puntos de tejido se están utilizando y cómo se están uniendo, y quiénes están promoviendo estas visiones y por qué. En este sentido, hay una necesidad urgente de considerar los movimientos y 
discursos eco-politizantes, como los de los movimientos políticos ambientales o los diversos indignados y otras movilizaciones políticas insurgentes que en los últimos años han estado exigiendo un nuevo proceso democrático constituyente. La articulación entre el pensamiento político-ecológico urbano y la democratización de las prácticas urbanas con una mirada a pensar si es que una forma de urbanización planetaria ecológicamente sensible, igualitaria, libre y solidaria todavía puede imaginarse para el siglo XXI es, a mi juicio, el mayor desafío intelectual para una ecología política urbana que desea ser políticamente performativa.

\section{Referencias}

Ali, S. H. \& Keil, R. (2008). Networked disease: emerging infections in the global city. Malden: Wiley-Blackwell.

Angelo, H. \& Wachsmuth, D. (2015). Urbanizing urban political ecology: a critique of methodological cityism. International journal of urban and regional research, 39(1), 16-27. https://doi. org/10.1111/1468-2427.12105

Armiero, M. (2014). Garbage under the volcano: the waste crisis in Campania and the struggles for environmental justice. En Armiero, M. \& L. Sedrez (Eds.). A history of environmentalism. Local struggles, global histories (pp. 167-184). London: Bloomsbury academic.

Bakker, K. (2003). An uncooperative commodity. Privatizing water in England and Wales. New York; Oxford: Oxford University press.

Bouzarovski, S. (2014). Energy poverty in the European Union: landscapes of vulnerability. WIREs energy and environment, 3(3), 276-289. https://doi. org/10.1002/wene. 89
Brechin, G. (2001). Imperial San Francisco: urban power, earthly ruin. Berkeley: University of California press.

Brenner, N. (2013) Implosions/Explosions: towards a study of planetary urbanization. Berlin: Jovis.

Buerk, R. (2006). Breaking ships: how supertankers and cargo ships are dismantled on the beaches of Bangladesh. New York: Chamberlain Bros.

Bulkeley, H. \& Betsill, M. (2005). Cities and climate change: urban sustainability and global environmental governance. London: Routledge.

Bullard, R. D. (1990). Dumping in Dixie: race, class, and environmental quality. Boulder: Westview.

Caprotti, F. (2014). Eco-urbanism and the eco-city, or denying the right to the city? Antipode, 46(5), 1285-1303. https://doi. org/10.1111/anti.12087

Coe, N., Kelly P., \& Yeung, H. (2007). Economic geography: a contemporary introduction. Oxford: Blackwell.

Cook, I. \& Swyngedouw, E. (2012) Cities, social cohesion and the environment: towards a future research agenda. Urban studies, 49(9), 1.959-1.979. https://doi. org/10.1177/0042098012444887

Cronon, W. (1991) Nature's metropolis: Chicago and the great west. New York: WW Norton and company.

Crouch, C. (2004) Post-Democracy. Cambridge: Polity press.

Davis, M. (2002). Dead cities. New York: The new press. 
Debord, G. (1994). The society of the spectacle. New York: Zone.

Freidberg, S. (2004). French beans and food scares: culture and commerce in an anxious age. Oxford: Oxford University Press.

Gandy, M. (2002). Concrete and clay: reworking nature in New York City. Cambridge, Massachusetts: MIT press.

Gandy, M. (2005). Cyborg urbanization: complexity and monstrosity in the contemporary city. International journal of urban and regional research, 29(1), 26-49. https://doi.org/10.1111/j.14682427.2005.00568.x

Graham, S. \& Marvin S. (2001). Splintering urbanism. London: Routledge.

Harvey, D. (1993). The nature of environment: dialectics of social and environmental change. In R. Milliband \& L. Panitch (Eds.). Real problems, false solutions. London: Merlin press.

Harvey, D. (1996) Justice, nature and the geography of difference. Oxford: Blackwell.

Heynen, N., Kaika, M., \& Swyngedouw, E. (2006). In the nature of cities: urban political ecology and the metabolism of urban environments. London: Routledge.

Heynen, N., Kurtz, H. E., \& Trauger, A. (2012). Food justice, hunger and the city. Geography compass, 6(5), 304-311. https:// doi.org/10.1111/j.1749-8198.2012.00486.x

Hillier, J. (2009). Assemblages of justice: The 'ghost ships' of Graythorp. international journal of urban and regional research, 33(3), 640-661. https://doi. org/10.1111/j.1468-2427.2009.00861.x
Intergovernmental Panel on Climate Change [IPCC]. (2013). Summary for Policymakers. In T. F. Stocker, D. Qin, G. K. Plattner, M. Tignor, S. Allen, J. Boschung, A., ... P. Midgley (Eds.). Climate change 2013: The physical science basis. (Working group I Contribution of to the fifth assessment report of the intergovernmental panel on climate change). Cambridge: Cambridge University Press.

Jameson, F. (2003). Future city. New Left Review, 5. Recuperado de https:// newleftreview.org/II/21/fredric-jamesonfuture-city

Kaika, M. (2005). City of flows: nature, modernity and the city. New York: Routledge. ISBN 10: 9780415947169. ISBN 13: 978-0415947169

Keil, R. (2003). Urban political ecology. Urban geography, 24(8), 723-738. https:// doi.org/10.2747/0272-3638.24.8.723

Keil, R. (2005). Progress report-urban political ecology. Urban geography, 26(7), 640-651. https://doi.org/10.2747/02723638.26.7.640

March,H.\& Saurí,D. (2013). The unintended consequences of ecological modernization: Debt-induced reconfiguration of the water cycle in Barcelona. Environment and planning A, 45(9), 2.064-2.083. https://doi. org/10.1068/a45380

Marquand, D. (2004). Decline of the public: the hollowing out of citizenship. Cambridge: Polity press. ISBN 10: 0745629105. ISBN 13: 978-0745629100

Marvin, S. \& Medd, W. (2006). Metabolisms of obecity: Flows of fat through bodies, cities, and sewers. Environment and planning $A, 38(2), 313-324$. https://doi. org/10.1068/a37272 
Masjuan, E., March, H., Domene, E., \& Saurí, D. (2008). Conflicts and struggles over urban water cycles: the case of Barcelona 1880-2004. Tijdschrift voor economische en sociale geografie, 99(4), 426-439. https:// doi.org/10.1111/j.1467-9663.2008.00477.x

Meadows, D. H., Meadows, D. L., Randers, J. \& Behrens III, W. W. (1972). The limits to growth: a report for the club of Rome's project on the predicament of mankind. New York: Universe books.

Monstadt, J. (2009). Conceptualizing the political ecology of urban infrastructures: Insights from technology and urban studies. Environment and planning A, 41, 1924-1942. https://doi.org/10.1068/a4145

Mostafavi, M. \& Doherty, G. (2010). Ecological urbanism. Harvard university graduate school of design. Baden: Lars Müller publishers. ISBN 10: 3037781890. ISBN 13: 978-3037781890

Mouffe, C. (2013) Agonistics. Thinking the world politically. London: Verso.

Njeru, J. (2006). The urban political ecology of plastic bag waste problem in Nairobi, Kenya. Geoforum, 37(6), 1.046-1.058. https://doi.org/10.1016/j. geoforum.2006.03.003

Pellow, D. (2007) Resisting global toxics. Transnational movements for environmental justice. Cambridge, Mass.: MIT press.

Rancière, J. (2000). La partage du sensible. Esthétique et polique. Paris: La Fabrique.

Robbins, P. (2007). Lawn people: How grasses, weeds, and chemicals make us who we are. Philadelphia: Temple University press.
Schlosberg, D. (2007). Defining environmental justice: Theories, movements and nature. Oxford: Oxford University press.

Swyngedouw, E. (1996). The city as a hybrid: on nature, society and cyborg urbanization. Capitalism nature socialism, 7(2), 65-80. https://doi. org/10.1080/10455759609358679

Swyngedouw, E. (2004). Social power and the urbanization of water: flows of power. Oxford: Oxford University press.

Swyngedouw E. (2006). Circulations and metabolisms: (Hybrid) Natures and (Cyborg) cities. Science as culture, 15(2), 105-121. https://doi. org/10.1080/09505430600707970

Swyngedouw, E. (2009). The antinomies of the postpolitical city: in search of a democratic politics of environmental production. International journal of urban and regional research, 33(3), 601-620. https://doi.org/10.1111/j.14682427.2009.00859.x

Swyngedouw, E. (2010). Apocalypse forever? Theory, culture \& society, 27(2-3), 213-232. https://doi. org/10.1177/0263276409358728

Swyngedouw, E. (2013a). Apocalypse now! Fear and doomsday pleasures. Capitalism, nature, socialism, 24(1), 9-18. https://doi.org/10.1080/10455752.2012.75 9252

Swyngedouw, E. (2013b). The nonpolitical politics of climate change. ACME. An international journal for critical geographies, 12(1), 1-8. Recuperado de https://www.acme-journal.org/index.php/ acme/article/view/948 
Swyngedouw E. (2014). Anthropocenic politicization: from the politics of the environment to politicizing environments. En J. Hedrén \& K. Bradley (Eds.). Green utopianism: Politics, practices and perspectives (pp.23-37). London and New York: Routledge.

Swyngedouw E. (2017). More-thanHuman constellations as immunological bio-political fantasy in the urbicene. En Gomez-Luque, M. \& G. Jafari (Eds.) New geographies 09: Posthuman (pp.20-28). New York: ActarD.

Swyngedouw, E. (2018). Promises of the political: Insurgent cities in a post-political environment. Cambridge, MA: MIT press.

Swyngedouw, E. \& H. Ernstson (2018). Interrupting the Anthropo-obScene: Immuno-biopolitics and depoliticizing ontologies in the anthropocene. Theory, culture \& society https://doi. org $/ 10.1177 / 0263276418757314$
Virilio, P. (1986). Speed and politics: an essay on dromology. New York: Semiotext.

Walker, G. (2009). Beyond distribution and proximity: exploring the multiple spatialities of environmental justice. Antipode, 41(4), 614-636. https://doi. org/10.1111/j.1467-8330.2009.00691.x

Walker, G. (2012). Environmental justice: concepts, evidence and politics. London: Routledge.

White, D. (2008). Bookchin. A critical appraisal. London: Pluto Press.

Williams, E. C. (2011). Combined and uneven apocalypse. Washington, D.C.: Zero books. ISBN: 978-1-84694-468-0

Žižek, S. (2008). Censorship today: Violence, or ecology as a new opium for the masses. [Blog]. Recuperado de http:// www.lacan.com/zizecology1.htm 